\title{
Didactique : une approche sociohistorique du concept
}

\author{
Yves Lenoir \\ Université de Sherbrooke, Québec, Canada
}

\section{Pour citer cet article :}

Lenoir, Y. (2020). Didactique : une approche sociohistorique du concept. Didactique, l(1), 12-39. doi : 10.37571/2020.0102

Résumé : L'article adopte une approche sociohistorique brièvement explicitée pour retracer l'émergence du concept de didactique. Il rappelle d'abord que le terme provient de la pensée grecque antique et qu'il a été porteur de significations distinctes, en fonction des contextes dans lesquels il était en usage. Après avoir évoqué sa mobilisation par Ratke et Comenius au $17^{\mathrm{e}}$ siècle, il retrace son retour en force dans la pensée éducative française à partir des années 1970 et avance une interprétation qui rejoint, croyons-nous, nombre de didacticiens. Enfin, l'article avance quelques pistes interprétatives du concept dans d'autres univers éducatifs et mentionne les fondements sociohistoriques qui ont conduit à ces différences de signification.

Mots-clés : didactique des disciplines, approche sociohistorique, origine du concept, interprétations diverses 


\section{Introduction}

Il nous est apparu pertinent, dans le cadre de ce premier numéro d'une nouvelle revue traitant des questions didactiques, de retracer, sans aucune prétention à l'exhaustivité, le processus sociohistorique d'émergence du concept de didactique. Non pas que des travaux antérieurs ne se soient pas déjà penchés sur cette question (par exemple, Artigue et Douady, 1986; Artigue, Gras, Laborde et Tavignot, 1994; Astolfi, 1990; Bronckart et Schneuwly, 1991; Brousseau, 2004; Develay, 1997; Jonnaert, 1991a; Lemoyne, 1996; Schneuwly, 1990a; Tasra, 2017), mais nous entendons rappeler son origine temporelle lointaine et esquisser différentes significations, sans cependant entrer dans les débats franco-français ce que ne permettent pas les contraintes éditoriales - qui se sont poursuivis depuis les années 1970 entre et dans les différentes disciplines d'enseignement ${ }^{4}$. En nous appuyant sur une approche sociohistorique que nous expliquerons brièvement au départ, nous proposerons ensuite une définition de la didactique des disciplines avant d'évoquer quelques-unes de ses conceptions véhiculées dans d'autres sociétés et de conclure en rappelant brièvement ce qui nous paraît être les fondements sociohistoriques des choix effectués en France et aux États-Unis.

\section{Une approche sociohistorique}

Notre formation en sociologie de la culture et en sociologie de la connaissance nous a conduit $^{5}$ à nous pencher sur les significations des notions et concepts en usage par l'entremise d'un processus sociohistorique fondé sur l'analyse des productions écrites actuelles et passées. Ainsi, dans le cadre de notre enseignement en didactique des sciences humaines et sociales d'abord, puis en fondements de l'éducation, et à travers nos recherches empiriques sur les pratiques d'enseignement au cours des 30 dernières années, nous avons cru en la nécessité d'une contextualisation temporelle et sociale des notions et

\footnotetext{
${ }^{4}$ Il n'est qu'à se rappeler qu'en 1993 est paru sous la direction de Desvé un Guide bibliographique des didactiques qui présentait au-delà de 1200 publications, avant tout d'auteurs français, portant sur la didactique et parues depuis son émergence dans la francophonie en 1970. Ou encore, la base de données francophone la plus importante, Francis, identifie pour la période 1995-2014 1802 publications, dont 40 livres. Nous avons, quant à nous, près de 500 livres sur la didactique dans notre base de données, ce qui montre qu'il n'existe aucune recension exhaustive récente. De plus, il n'est pas une publication (livre, article scientifique) dans le champ de l'éducation dans la francophonie (Belgique francophone, France, Luxembourg, Québec, Suisse romande) portant sur l'enseignement des disciplines scolaires ou la formation à l'enseignement des disciplines scolaires qui ne se réfère pas d'une manière ou d'une autre au concept de didactique. Pour un panorama succinct de l'évolution des conceptions et courants didactiques en France, voir par exemple Simoneaux et Simoneaux (2014).

${ }^{5}$ Veuillez noter que tout au long de ce texte nous utilisons le nous de modestie. En conséquence, parce que le pronom nous ne désigne qu'une seule personne, l'adjectif ou le participe qui s'y rapporte se met au singulier. Il s'accorde en genre selon le sexe de la personne qu'il désigne. L'accord se fait aussi au singulier pour l'adjectif même (nous-même) lorsqu'il est juxtaposé au pronom.
} 
concepts structurant nos activités intellectuelles. Une telle option repose sur les trois arguments suivants.

- Premièrement, d'un point de vue historique, nous considérons avec Bloch (1977/1949) que « l'incompréhension du présent naît fatalement de l'ignorance du passé » (p. 47), mais, ainsi qu'il l'ajoutait, « il n'est peut-être pas moins vain de s'épuiser à comprendre le passé, si l'on ne sait rien du présent » (Ibid.).

- Deuxièmement, d'un point de vue épistémologique, nous retenons que l'observation des faits empiriques n'a de sens et de pertinence que dans la mesure où elle s'inscrit à la fois dans un processus de (re)construction théorique et d'une nécessaire construction de la rupture par laquelle la production de l'ensemble conceptuel est elle-même interpellée et ne se substitue pas à l'analyse. Une distanciation critique s'impose en conséquence entre les moments de conceptualisation, d'observation et d'analyse, de manière à offrir des espaces de liberté permettant les réaménagements, car ni le cadre conceptuel ni l'observation empirique, indispensables cependant, ne disent la réalité effective.

- Troisièmement, d'un point de vue social, ainsi que nous l'exposons ailleurs dans le détail (Lenoir, 2017), nous suivons Freitag (1986) qui, à travers l'élaboration d'une théorie du symbolique, questionne les conditions de production de la connaissance en tant que pratique sociale faisant obligatoirement appel à des médiations comme modes de régulation des rapports sociaux. Les trois fonctions (empirique, théorique, opératoire) du rapport d'objectivation scientifique, rappelle-t-il, ne s'autonomisent pas les uns face aux autres, mais elles s'inscrivent dans un rapport dialectique où la fonction théorique, qui est « porteuse en même temps de la mémoire et de l'intentionnalité » (p. 221), assume le rôle central de médiation synthétique dans le rapport d'objectivation scientifique. De plus, cette démarche ne peut faire fi ni du processus d'attribution de significations symboliques à l'agir humain en fonction des normes sociales, de l'idéologie dominante, de son ancrage dans le réel (la fonction de valeur), ni de la réinscription de la signification symbolique (de la réalité construite) dans la temporalité sociohistorique (en tant que cas particulier du possible actualisé) qui assure la possibilité d'une intersubjectivité (la fonction de sens). Notons que ces différentes fonctions, incluant celles de sens et de valeur, ne peuvent guère être exposées dans ce texte. 
Sur la base entre autres de ces fondements (processus génétique, social et épistémologique) ${ }^{6}$, nous avons, comme nous l'avons fait par exemple pour les notions d'interdisciplinarité (Lenoir, Rey et Fazenda, 2001; Lenoir et Sauvé, 1988a, 1988b) ou de finalités éducatives (Lenoir, Adigüzel, Lenoir, Libâneo et Tupin, 2016), recensé et analysé (en nous référant systématiquement à la dimension historique) au cours des années dans le cadre de nos recherches, avec le concours de nos assistants de recherche et de collègues associés à notre centre de recherche, puis à la chaire de recherche du Canada sur l'intervention éducative, et sur la base de grilles d'analyse reposant sur les cinq fonctions ci-haut mentionnées, les diverses publications sur les didactiques (générale, des disciplines) dans l'univers francophone, mais aussi anglophone, germanique, hispanique, scandinave et lusophone, en nous centrant sur les sources documentaires de la deuxième moitié du $20^{\mathrm{e}}$ siècle et sur celles relatives au $17^{\mathrm{e}}$ siècle, choix que la suite de l'article permet de comprendre. Plusieurs travaux témoignent des résultats obtenus (Chené, Gauthier, Martineau, Dolbec, Lenoir, Gaudet et Charland, 1999; Favre et Lenoir, 2015; Lebrun et Lenoir, 2004; Lenoir, 1993, 2000; Lenoir, Hasni et Froelich, 20157). À une attitude d'interrogation critique ${ }^{8}$ se marie une approche comparative reposant sur «le modèle intégral » où "toutes les unités de comparaison sont traitées avec le même niveau d'égalité » (Lenoir, Esquivel et Jean, 2020, p. 30). Une approche de type qualitatif, « critique et théorique axée sur les contenus [...] multidiscursive et multilingue » (Vigour, 2005, p. 136-137), a ensuite été mise en œuvre en s'appuyant sur l'approche sociohistorique.

\section{Les origines du mot}

Le mot didactique émane de la pensée grecque. En grec ancien, «J'enseigne, j'instruis »

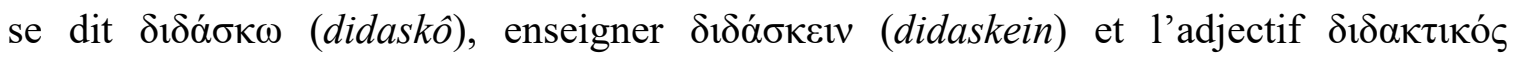

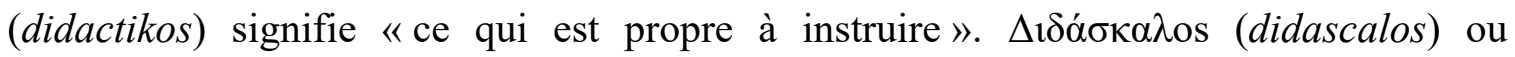
$\gamma \rho \alpha \mu \mu \alpha \tau o \delta เ \delta \alpha ́ \sigma \kappa \alpha \lambda o s$ (grammatodidascalos), c'est «l'instituteur, le maître d'école qui apprend à lire [...] $\delta 1 \delta \alpha \sigma \kappa \alpha \lambda \varepsilon i ̈ o v$, c'est l'établissement où il enseigne » (Marou, 1965/1948,

\footnotetext{
${ }^{6}$ Pour une présentation exhaustive des fondements qui sous-tendent nos travaux de recherche, voir Lenoir (2011, 2019a) pour une vue sommaire et Lenoir (2017) pour un exposé extensif.

${ }^{7}$ Plusieurs éléments de cet article sont extraits d'un article paru aux États-Unis dans la Revue Issues in Interdisciplinary Studies (Lenoir, Hasni et Froelich, 2015), article qui présentait deux conceptions de l'interdisciplinarité dans l'éducation scolaire primaire et secondaire (elementary et middle schools), l'une anglosaxonne et étatsunienne reposant sur la notion de curriculum, l'autre francophone et européenne basée sur la notion de didactique.

${ }^{8}$ On pourrait croire que l'exigence d'une pensée critique est d'usage relativement récent. Pourtant, on la retrouve de manière explicite au $12^{\mathrm{e}}$ siècle dans le Sic et non d'Abélard (1851/土 1120) qui s'inspire lui-même d'Aristote. Abélard défend non un scepticisme rationnel, mais l'importance du travail rationnel de l'esprit : «Étant donné que c'est la première clé de la sagesse, cela exige une attitude fréquente d'interrogation [...]. Doutant cependant de nos capacités à mener une enquête, nous devons enquêter sur nous-même pour percevoir la vérité » (p. 16-17; notre traduction du latin).
} 
p. 221). Divers autres termes sont dérivés ${ }^{9}$ dont celui de didascalie, issu du grec (didaskalia), introduit au $18^{\mathrm{e}}$ siècle en français pour caractériser des instructions, des notices portant sur la mise en scène données par un auteur de pièce de théâtre aux acteurs. Ce dernier mot, toutefois, est repéré dans 21 versets de la Bible, selon la traduction du grec de Louis Segond (1880), pasteur protestant et théologien français du $19^{\mathrm{e}}$ siècle, avec le sens de préceptes, surtout de doctrine, mais aussi d'enseignement, d'instruction. Repris par la tradition chrétienne dans le sens d'un enseignement portant sur la discipline ecclésiastique issu de celui des apôtres, il se retrouve dans La didascalie des Apôtres (Nau, 1902) dont il ne reste qu'une traduction syriaque du grec original produit au commencement du $3^{\mathrm{e}}$ siècle. Elle serait inspirée - ce que rejette Nau (Ibid.) dans l'introduction de La didascalie - d'un manuscrit

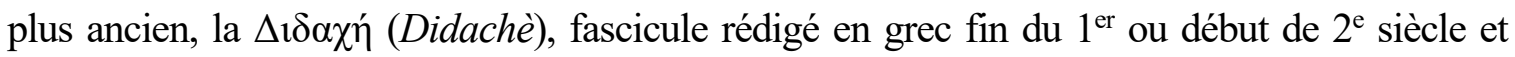
intitulé Doctrine du Seigneur transmise aux nations par les douze apôtres.

Quoi qu'il en soit, il ne fait donc aucun doute que tant l'adjectif que le substantif ont été utilisés dans les premiers temps de l'Église chrétienne dans un esprit prescriptif pour énoncer sa doctrine, son enseignement et, note Marrou (1965/1948), la formation du catéchumène. L'adjectif était en usage dans l'Antiquité grecque, mais aussi romaine (de Caussade, 1719), où différentes significations lui étaient attribuées en fonction du style poétique (l'examètre dactylique), de la visée poursuivie par divers auteurs grecs et romains ${ }^{10}$. La notion se retrouve également à Byzance où Justinien ferme en 529 les écoles d'Athènes pour les transférer à Constantinople et les mettre sous contrôle impérial en tant

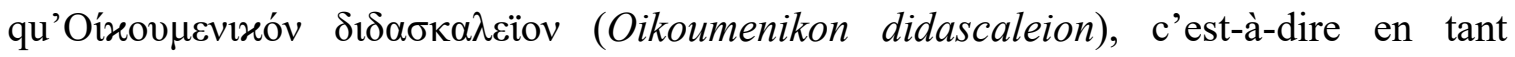
qu'écoles des hautes études « dirigées par un collège de douze didascales » (d'Irsay, 1933, p. 228) et qui subsistèrent jusqu'à la conquête ottomane en 1453. Lemerle (1971) montre combien ces termes étaient abondamment utilisés dans ces écoles byzantines en tant qu'héritage de la Grèce ancienne. Encore au 12 $2^{\mathrm{e}}$ siècle, Hugues de Saint-Victor (1961/av. 1137) publie une œuvre de très grande importance pour l'époque, le Didascalicon qui est considéré comme « un véritable traité des études, embrassant tout l'ordre du savoir » (Paré, Brunet et Tremblay, 1933, p. 94) et qui présente les sept arts, trivium et quadrivium, de manière rigoureuse et en expose les modes d'enseignement. Enfin, l'adjectif aurait été introduit en français par de Maumont en 1534. En français également, le substantif didactique se retrouve beaucoup plus tardivement dans les éditions de 1955 du Robert et de 1960 du Littré où il est défini comme l'art d'enseigner. Bref, le terme emprunté au grec et portant sur l'action formatrice n'a pu être oublié dans les milieux religieux nourris de latin et de grec jusqu'à l'époque de sa première renaissance au $12^{\mathrm{e}}$ siècle.

\footnotetext{
${ }_{9}^{9}$ Par exemple, si l'autodidacte désigne celui qui s'est instruit lui-même, le didactisme est un terme porteur d'un sens péjoratif, traduisant une expression pompeuse, un discours ennuyant, fastidieux ou péremptoire.

10 « La poésie didactique [...] a pour objet d'enseigner des vérités de l'ordre moral ou physique, dans les arts et dans les sciences. [...] Son essence est l'enseignement de préceptes » (de Caussade, 1719, p. 103).
} 


\section{Le relais du $17^{\mathrm{e}}$ siècle}

Il est d'usage, chez les didacticiens francophones, comme le mentionne Schneuwly (1990a) qui date de $1613^{11}$ la naissance du terme didactica, de se réclamer d'un père fondateur lointain, Ratke ou Ratichius (1571-1635) en latin, lequel prônait une Methodus didactica, un nouvel art de l'enseignement. Comenius (1592-1670) reprend dans sa Didactica magna (1657/1952) les idées de son maître. Pour Hopmann (1992) et Gundem (1992), qui présentent le courant didactique germano-nordique, on peut remonter à ces deux pédagogues du $17^{\mathrm{e}}$ siècle qui « ont fourni une théorie de l'apprentissage, basée sur les stades naturels de développement. Finalement, l'enseignement doit être conscient à la fois du contenu et de l'apprenant [...]. Cette tripartition [sur laquelle nous allons revenir] a été un concept central dans le développement de la didactique » (Hopmann, 1992, p. 4), ce que nous aborderons plus loin. Cependant, « ce n'est seulement que depuis les années 1920 [...], que le contenu de l'enseignement scolaire, le curriculum, et non les méthodes qui sont devenues le point focal de la didactique »(Gundem, 1992, p. 62) ${ }^{12}$.

Ainsi que le relève Schneuwly (1990a), Comenius propose, en faisant appel aux lois de la nature, une méthode d'enseignement général et des méthodes spécifiques pour chaque discipline d'enseignement. Et «didactica est un terme de combat» (p. 22) introduit par Ratke pour lutter «contre le féodalisme et pour l'unité nationale allemande » (Ibid.). L'introduction du terme didactique dans son discours éducatif a ainsi traduit au $17^{\mathrm{e}}$ siècle une volonté de transformation sociale d'importance en un temps qui fut pour les Tchèques, mais aussi pour nombre d'Européens, un âge des ténèbres (Denis, 1994) durant lequel, selon l'expression de Servier (1967), a sévi une nouvelle visite en Europe des Cavaliers de l'Apocalypse (les guerres de religion et la peste). Il faut aussi se rappeler que Comenius fut un membre du mouvement protestant des Frères Moraves, mouvement lié aux doctrines hussites. Lors de la guerre de Trente Ans, il doit fuir son pays, sous la menace des troupes catholiques espagnoles, en abandonnant épouse et enfants qui moururent de la peste. Il doit fuir à nouveau la Pologne pour se réfugier finalement à Amsterdam. Les travaux francophones contemporains (par exemple Cauly, 1995; Canavolas et Bibeau, 1996; Denis, 1994) soulignent l'importance de son œuvre sur deux plans : son caractère révolutionnaire qui prône une éducation de meilleure qualité et qui permettrait, à ses yeux, de s'opposer

11 À l'encontre de Schneuwly (1990a), nous venons de rappeler l'ancienneté grecque et latine du mot didactique et de quelques-uns de ses dérivés. On ne peut donc attribuer à l'année 1613 la naissance du terme. ${ }^{12}$ Il faut comprendre ici méthodes non dans le sens des démarches à caractère scientifique, mais dans celui de techniques, de procédures à appliquer. Rappelons que Bru (2006) montre combien les méthodes en pédagogie sont bien davantage porteuses d'illusions au regard de leur efficacité, le plus souvent légitimée par des discours dogmatiques, et il souligne la grande prudence à adopter à leur égard. 
aux conceptions catholiques; un caractère visionnaire qui défendait la mise en place d'une science rationnelle universelle.

Mais à trop vouloir trouver une origine lointaine en ne s'en tenant qu'au mot lui-même, ne risque-t-on pas, comme l'a bien montré par exemple Benvéniste (1969) avec Le vocabulaire des institutions indo-européennes, d'introduire un anachronisme, source d'une profonde confusion sémantique et de masquer ainsi tout particulièrement les enjeux sociaux d'une époque donnée, la nôtre en ce cas?

\section{La résurrection au début des années 1970}

Après une période de quelque deux siècles pendant lesquels l'attention a été avant tout portée - avec la montée de l'indivualisation et l'émergence de la notion d'enfant (Shorter, 1977; Snyders, 1965) dans le contexte du capitalisme bourgeois triomphant - sur les dimensions pédagogiques et les caractères bio-psycho-physiques des enfants, ce dont témoignent explicitement les pères fondateurs des sciences de l'éducation en France (Lenoir, 2019b), le concept de didactique revient à la surface, d'abord en Allemagne note Schneuwly (1990a) en tant qu'une discipline devenant progressivement autonome par rapport à la pédagogie qui « porte aussi bien sur la définition des objectifs généraux, sur le choix des contenus et sur l'organisation de l'école que sur l'élaboration des programmes d'études et des méthodes d'enseignement» (p. 23). Il s'agit alors d'une didactique générale.

Selon Dorier, Leutenegger et Schneuwly (2013), la réintroduction du concept de didactique dans la francophonie, au tournant des années 1970, découle de trois facteurs principaux largement distincts de ceux du $17^{\mathrm{e}}$ siècle $^{13}$. Les deux premiers sont d'ordre institutionnel; le troisième relève d'enjeux socio-éducatifs et culturels. L'émergence des didactiques des disciplines comme champs disciplinaires découle, premièrement, du phénomène de massification qui a marqué l'enseignement secondaire à partir des années 1950. Cette arrivée massive d'élèves a conduit à une refonde en profondeur des plans d'études de toutes les matières scolaires, puis à une réforme de la formation des enseignants. C'est aussi ce que soulignent Astolfi (1990) et Brousseau (2004). Deuxièmement, la suppression des écoles normales pour une universitarisation de la formation des enseignants ${ }^{14}$ a suscité des

\footnotetext{
13 Artigue et Douady (1986), par exemple, retiennent pour leur part l'apport des trois facteurs suivants : l'épistémologie génétique piagétienne; la notion d'obstacle épistémologique; l'école genevoise de psychologie sociale.

${ }^{14}$ Cette universitarisation a d'abord eu lieu au Québec dès 1969. Toute formation initiale et continue à l'enseignement créditée au primaire et au secondaire est assurée dans des facultés d'éducation par des enseignants chercheurs détenant obligatoirement un doctorat. La France a créé en 1991 les instituts universitaires de formation des maîtres (IUFM) qui sont devenus en 2013 des écoles supérieures du
} 
rapprochements obligatoires de la part des différentes professions enseignantes, ce qui a conduit à la production « de disciplines académiques articulant recherche et formation pour les enseignants » (Dorier, Leutenegger et Schneuwly, 2013, p. 10). Il importe aussi de reconnaître que le succès premier du terme didactique est lié au statut qu'il permettait aux formateurs d'enseignants de s'attribuer dans l'espace universitaire, a priori non rattachés à une structure disciplinaire, ce que la didactique leur offrira. Ce n'est peut-être pas un hasard si ce sont les mathématiciens, devenus des formateurs, qui s'en sont emparés les premiers $^{15}$. Le troisième facteur réside dans la réaction à des théories et à des pratiques qui, sans oublier les objets de savoir à enseigner - car cela ne se peut, ainsi que le soulignent Astolfi (1997) et Houssaye (1997), ou encore Develay (1997) -, les avaient toutefois mis entre parenthèses en s'appuyant sur des courants pédagogiques centrés sur les activités d'éveil ${ }^{16}$. À cela s'ajoutait une critique croissante au regard des sciences de l'éducation «jugées trop déconnectées des questions pratiques, à cause des théories de référence d'alors » (Develay, 1997, p. 60). Ces courants porteurs du concept de didactique en construction et centrés sur les processus de formation des enseignants s'insurgeaient contre le didactisme (au sens américain du terme) qui caractérisait les méthodes traditionnelles d'enseignement et les conceptions de l'apprentissage que celles-ci sous-tendaient. Ils s'insurgeaient surtout contre l'importance accrue accordée aux aspects pédagogiques qui tendaient, à leurs yeux, de minimiser l'importance des apprentissages des contenus cognitifs des différentes disciplines scolaires ${ }^{17}$. Il faut aussi se rappeler une certaine

professorat et de l'éducation (ESPE). La Suisse, au début des années 1990, et la Belgique, en 1995, ont créé les hautes écoles universitaires. Toutes ces formations des enseignants sont professionnalisantes et se caractérisent par un référentiel de compétences.

15 Nous avons montré, à la suite d'une recension critique d'auteurs surtout français et anglais, l'existence de quatre configurations disciplinaires dans le système d'enseignement, dont la discipline scolaire en tant que prolongement de la discipline scientifique (ou la dimension disciplinaire), les mathématiques en étant le cas le plus fort et le plus évident (Hasni, 2001; Lenoir et Hasni, 2006).

${ }_{16}$ Par activités d'éveil, il faut entendre ces propositions et démarches pédagogiques qui, dans un souci de démocratisation du système éducatif français, « étaient, au moment de leur genèse, à partir des années 1960-1965, destinées à donner une orientation pédagogique forte à l'école élémentaire dans un ensemble de mesures désigné par le terme de rénovation pédagogique » (Best, 2006, p. 48). Plusieurs publications sont parues à cette époque (par exemple : Best, 1973; Serri, 1977) dont, tout au long des années 1970, un ensemble de numéros thématiques de la revue Recherches pédagogiques (par exemple : $\mathrm{n}^{\circ} 51,62,70,93$ ) émanant de l'Institut national de recherche et de documentation pédagogiques (INRDP, 1971, 1973, 1974, 1978).

17 Il s'agissait en particulier d'une opposition, d'une part, aux courants de l'école active et, d'autre part, des conceptions pragmatiques que prônaient les activités d'éveil. Celles-ci étaient inspirées principalement du mouvement herbartien, puis de Dewey, et du courant humaniste - Rogers, Bany et Johnson, etc. - provenant des États-Unis, mais aussi de l'École nouvelle (Montessori, Freinet, Decroly, etc.) et de la pédagogie ouverte au Québec (Paquette, 1976, 1985; Paré, 1977). Dans la perspective alors développée en France, mais aussi au Québec, les activités d'éveil concevaient l'école comme un milieu de vie où l'enseignement était centré sur des questions de la vie quotidienne et sur les expériences de vie, ainsi que sur des activités visant l'épanouissement de l'enfant. Dès lors, l'accusation était claire à leur égard : on n'enseigne plus dans les écoles; on a oublié l'existence des savoirs disciplinaires et on se complaît dans une attention toute tournée vers le sujet et son épanouissement comme être humain. Bref, trop de socialisation, pas assez d'instruction ! 
réaction, pas nécessairement consciente de la part des didacticiens, contre la montée de l'utilitarisme qui dominera les logiques éducatives à partir des années 1990 à travers un néolibéralisme triomphant et qui tend à réduire l'instruction à une préparation d'un capital humain à l'emploi (Lenoir, Adigüzel, Lenoir, Libâneo et Tupin, 2016).

Ce troisième facteur est capital. En effet, la recréation du mot didactique et son retour en force dans le champ de l'éducation scolaire francophone, ainsi que le rappellent par exemple Develay (1997), Raisky (1996) et Schneuwly (1990a), adoptait une posture critique et contestataire qui pouvait, à première vue, être rapprochée de l'attitude coménienne. Autour des années 1970, les premiers didacticiens francophones ont adopté une orientation revendicatrice en vue d'introduire des changements profonds dans la conception du rapport enseignement-apprentissage, mais leurs revendications reposaient sur des motifs bien différents, ni politiques, ni religieux. Chez Comenius, la didactique ouvrait assurément à une position avant-gardiste par le souci de promouvoir une éducation plus démocratique et émancipatrice qui puisait dans la tradition hussite (Capková, 1992; Delumeau, 1965). Cette tradition soutenait des visées d'actualisation d'une doctrine profondément engagée dans la société (Garin, 1968). Dans la francophonie, à l'orée des années 1970, les premiers chercheurs ont remis la question de la didactique à l'ordre du jour, en lui gardant « une connotation combative et critique » (Schneuwly, 1990b, p. 217). Ils ont voulu réaffirmer l'importance du savoir scientifique et de son appropriation cognitive comme dimensions émancipatrices en éducation, dans la tradition républicaine française. Ces chercheurs ont voulu également réaffirmer le caractère profondément dialectique du rapport enseignement-apprentissage, car ce rapport exige un changement de perspective épistémologique et psychologique. À la posture néopositiviste et néobéhaviorale qui dominaient des années 1950 à la fin des années 1970, ou à la vision à tendance humaniste des fondateurs des sciences de l'éducation (Lenoir, 2019b), se sont substituées des conceptions psychologiques et épistémologiques à tendance constructiviste. Au lieu de concevoir le savoir comme un donné a priori, préexistant, inscrit dans le discours écrit, dans le manuel scolaire en particulier, les didacticiens francophones ont adopté une autre conception, issue de la tradition dialectique qui met en avant le processus fondamental de la production sociale, historiquement et spatialement datée, de la réalité : le savoir est le produit temporaire et limité de l'action humaine et sociale en contexte qui exprime une représentation symbolique du monde réel (Freitag, 1986; Lenoir, 2017) ${ }^{18}$. Morf, Grize et Pauli (1969) et Morf (1972), disciples de Piaget, sont peut-être les

\footnotetext{
${ }^{18}$ Cette perspective dialectique, reprise dans la conclusion de cet article, est au fondement de la notion de tripartition (sujet, objet, enseignant) sur laquelle repose le triangle didactique, notion parfois profondément dévoyée (Favre et Lenoir, 2015). Sur les dangers de dérives qu'elle peut susciter, voir également Halté (1992), Jonnaert (1991), Meirieu (1985, 1987). Les différentes dérives (épistémologique, psychologique, pédagogique) résultent fondamentalement, selon la logique du paradigme de la simplification, d'une volonté de réduction des variables à considérer dans le processus enseignement-apprentissage. Plutôt que d'adopter
} 
premiers à avoir mis en exergue cette perspective dialectique revendiquée par les didactiques $^{19}$. Morf, Grize et Pauli (1969) proposent «une théorie générale des interventions sur la pensée et sur son fonctionnement» qui s'appuierait sur trois axes interreliés : l'axe psychologique, relatif au sujet; l'axe épistémologique, qui se réfère à l'objet de la connaissance; l'axe logique « qui doit permettre l'analyse des rapports en jeu et la mise en œuvre de stratégies convenables » (p. 25) ${ }^{20}$ et qui se réfère à l'enseignant.

Cette nouvelle orientation, que véhiculait la didactique, où se mêlait le débat sur le statut de la didactique comme discipline universitaire, par là sur le statut des formateurs - débat déjà mentionné précédemment et que relève également Brousseau (2004) -, a été mise en avant au départ (dans les années 1970 et 1980) surtout par les mathématiciens qui faisaient alors la promotion de l'enseignement des mathématiques nouvelles. Toutefois, au-delà de ces quelques points d'accord, auxquels il faudrait ajouter l'adhésion fondamentale de la plupart des didacticiens, pour différentes raisons - institutionnelles, idéologiques, politiques, etc. - impossibles à présenter ici, relatives entre autres aux découpages disciplinaires relevant du système des sciences, différentes conceptions de la didactique ont foisonné (Lenoir, 2000), dues entre autres à plusieurs angles d'approche du concept ${ }^{21}$

une perspective dialectique en prenant en compte les différentes composantes, le paradigme de la simplification retient le principe de la causalité linéaire, ce qu'illustrent bien les écrits de Houssaye (1988, 1994, 1997) avec la notion de tiers exclu qui, il est vrai, vient faire le fou, et que stigmatisent par exemple Halbwachs (1971) ou Piaget (1971). Des visions réductrices du triangle didactique (ou système didactique) s'observent aussi, par exemple, chez Legendre (1983) où la dimension didactique n'apparaît pas explicitement, étant noyée dans la fonction d'agent.

${ }_{19}$ Les influences principales sont certes piagétiennes (Piaget, 1967, 1971), mais aussi de plus en plus, sinon surtout de nos jours, vygotskyiennes. Ses fondements contemporains se trouvent tout particulièrement dans les conceptions dialectiques que l'on retrouve chez Hegel, Marx, Piaget (Lenoir, 2017), mais aussi chez Moscovici (1970) et Habermas (1973, 1976) par exemple, puis chez Le Moigne (1984) et Morin (1977).

${ }^{20}$ Ainsi que nous l'avons mis en évidence (Lenoir, 2017), ces auteurs s'inspirent directement d'Habermas (1973, 1976). Celui-ci «adopte la typologie épistémologique suivante : le pôle ontologique fait référence aux sciences historico-herméneutiques, compréhensives, qui relèvent d'un intérêt de connaissance d'ordre pratique; le pôle fonctionnel renvoie aux sciences empirico-analytiques, descriptives, qui procèdent d'un intérêt de connaissance d'ordre technique (instrumental); le pôle génétique, quant à lui, renvoie aux sciences critiques qui procèdent d'un intérêt de connaissance d'ordre émancipatoire » (Lenoir, 2017, p. 122). Morf, Grize et Pauli (1969) sont également influencés par Le Moigne (1984) qui s'appuie sur Piaget. Pour Le Moigne, ainsi que nous le rappelons (Lenoir, 2017), « la définition d'un objet, qu'il qualifie de modélisation, requiert une triangulation reliant les perspectives ontologique (ce qu'est l'objet), fonctionnelle (ce que fait l'objet) et génétique (ce que devient l'objet). Il rapproche également ces trois modes de modélisation de la triade Phéno - Ego - Géno de Morin (1977, 1980)» (p. 122-123), mais aussi de «l'interprétation contemporaine de Jean Piaget restaurant les épistémologies constructivistes et génétiques, en fondant sur l'interaction Sujet - Objet [...] le mode privilégié de construction des représentations et donc des connaissances » (Ibid., p. 123).

${ }^{21}$ Nous avions alors distingué (Lenoir, 2000) quatre angles d'approche : selon les secteurs d'activité des praticiens et des didacticiens universitaires; selon les situations professionnelles des acteurs; selon les objets; selon les finalités poursuivies (recherche, formation, enseignement). 
et aux différentes conceptions de ses finalités : une science des procédés ${ }^{22}$; un ensemble (une didactique générale) de processus d'appropriation des savoirs en s'appuyant sur des théories d'origine psychologique essentiellement piagétiennes (exemple : Aebli, 1951; Dienes, 1964; Marquez, 1960) ${ }^{23}$; une discipline pleinement scientifique (exemple: Margolinas, 1993); une didactique axée sur la recherche-action, la plus répandue de nos jours. Chacune de ces quatre conceptions est fondée sur un registre distinct : technoinstrumental, psychologique, épistémologique, praxéologique (Bronckart, 1989; Lenoir, 2000).

Une illustration des débats de l'époque se retrouve en français, alors que les linguistes ont rapidement suivi le mouvement initié par les mathématiciens en voulant développer une didactique du français fondée sur la linguistique (à une époque où dominait la pensée structuraliste en France). Des publications de Chiss $(1985,1989)$ et de Galisson (1985, 1986, 1990) témoignent de cette tentative. De plus, dans un effort de clarification des positions entre une théorisation didactique a priori du savoir disciplinaire s'opposant à une didactique qui se présenterait comme théorisation de la pratique pédagogique, entre la recherche de sens s'opposant à celle de la fonctionnalité, certains chercheurs ont proposé de distinguer didactique et didaxologie (De Corte, Geerligs, Lagerweij, Peters et Vandenberghe (1979) ou didactique et didactologie (Galisson, 1985, 1986, 1990). Puis, tous les chercheurs dans les autres disciplines d'enseignement se sont mis progressivement à adhérer au concept de didactique.

\section{Une interprétation du concept}

Que faut-il alors entendre par didactique des disciplines? Bien entendu, il existe de nombreuses définitions ${ }^{24}$, mais il nous paraît possible d'extraire d'un ensemble d'auteurs cités ci-dessous des caractéristiques largement partagées par la communauté des didacticiens, et plus largement, par la communauté des chercheurs en éducation. Les

22 «"Ensemble des méthodes, techniques et procédés pour l'enseignement [...]. La didactique met principalement l'accent sur les moyens d'enseigner, sur le comment faire", écrivait Mialaret (1979, p. 159-160) en reprenant la représentation dominante encore à l'époque qui faisait de la didactique une science auxiliaire de la pédagogie » (Lenoir, 2000, p. 186).

23 «La didactique scientifique se donne pour tâche de déduire de la connaissance psychologique des processus de formation intellectuelle les mesures méthodologiques les plus aptes à les provoquer » (Aebli, 1966, p. 2).

24 Au-delà des débats déjà précédemment mentionnés, nous pouvons signaler, à titre illustratif, les conceptions avancées par Avanzini (1987) ou par Delorme (1986) qui privilégient une didactique générale ou interdisciplinaire, par Johsua (1999) et Sachot (1997), le premier dénonçant l'absence de perspective sociale, le second la non-reconnaissance de l'ancrage sociohistorique et culturel du système didactique, ou encore par Aebli (1951) et Marquez (1960) qui adoptent un modèle directement lié à la psychologie génétique. 
didactiques des disciplines sont des disciplines de recherche « qui analysent les contenus (savoirs, savoir-faire...) en tant qu'ils sont objets d'enseignement et d'apprentissage » (Reuter, 2010, p. 69) et qu'ils se retrouvent dans les disciplines scolaires. Les didactiques se distinguent ainsi des autres disciplines qui, dans le champ de l'éducation,

- analysent les contenus mais sans se préoccuper de l'enseignement ou des apprentissages (par exemple les mathématiques, la linguistique, la biologie, l'histoire...);

- analysent l'enseignement ou les apprentissages mais en ne se centrant pas sur les contenus (par exemple la pédagogie, la philosophie, la sociologie ou la psychologie de l'éducation...) (p. 69).

Les didactiques des disciplines sont alors saisies comme le travail à visée opératoire de problématisation des contenus de savoir nécessaires à l'atteinte des finalités éducatives : elles posent, dans chaque discipline, les problèmes de la production du savoir, de la variation des contenus cognitifs, de l'organisation et de la sélection de ces savoirs au niveau scolaire en vue de leur mise en œuvre dans l'enseignement et de leur aménagement pour respecter les capacités des élèves. Elles questionnent la nature même de la discipline enseignée, ses contours épistémologiques et le sens des contenus cognitifs à enseigner, et réfléchissent à leur aménagement, toujours du point de vue cognitif, pour les rendre accessibles aux élèves. Dit autrement, elles étudient les phénomènes de mise en circulation des connaissances disciplinaires et elles théorisent les phénomènes d'enseignement de chaque discipline d'enseignement. Suivant Astolfi et Develay (1989), Audigier (1988), Brousseau (1986), Cornu et Vergnioux (1992), ou encore Bronckart et Schneuwly (1991), Chevallard (1985) et Vergnaud (1983) et nombre d'autres didacticiens, la didactique se définit certes comme une discipline de recherche - ce qui est explicitement énoncé par Balacheff et Laborde (1992) ${ }^{25}$ - fondée rationnellement sur des bases épistémologiques et théoriques solides; mais elle est aussi centrée sur l'étude de contenus cognitifs exclusifs ceux de chaque discipline dans son autonomie vue comme indispensable. Par ailleurs, elle ne propose des situations d'enseignement que dans la mesure où ces dernières sont attestées par un contrôle expérimental serré au moyen d'ingénieries didactiques. Dès lors, penser les didactiques requiert l'établissement d'interactions indissociables entre les composantes du système qui les constitue.

\footnotetext{
${ }^{25}$ Pour Balacheff et Laborde (1992), « la recherche en didactique a pour principal objet d'étude les conditions de la construction des connaissances complexes par des sujets humains, apprenants enfants ou adultes, dans des situations délibérément organisées à cette fin (situations d'enseignement ou de formation). L'originalité de ces recherches réside dans la prise en compte, tant au niveau méthodologique que théorique, de la spécificité des connaissances en jeu » (p. 69). Cette définition met l'accent sur le fait qu'il existe des points communs et des caractères spécifiques à chacune des disciplines enseignées.
} 
Les didactiques des disciplines ne peuvent donc être confondues ni avec les pratiques d'enseignement et d'apprentissage dans chaque discipline scolaire, ni avec les prescriptions qui proviennent des lois ou des instructions officielles, des manuels scolaires, etc., ni avec les recommandations qui encadrent les pratiques d'enseignement et qui proviennent de multiples sources (inspecteurs, conseillers pédagogiques, associations et syndicats enseignants, etc.). Elles sont distinctes des approches pédagogiques qui relèvent du travail des chercheurs en pédagogie ${ }^{26}:$ «Elles doivent ainsi mener des luttes sur deux fronts au moins : par rapport au pédagogique et par rapport aux disciplines académiques de référence » (Dorier, Leutenegger et Schneuwly, 2013, p. 16). Elles reposent sur une structure conceptuelle relativement partagée dans sa généralité par la communauté des didacticiens (par exemple, la transposition, la dévolution, la théorie des situations didactiques, les pratiques de référence, le modèle tripolaire, etc.). À titre illustratif, les didacticiens ont retenu un modèle général de référence qui a été qualifié de système didactique. Le système didactique consiste en l'interaction entre trois sous-systèmes qui implique une relation didactique entre sujet (les élèves), objet (les contenus d'enseignement) et enseignant. Il provient d'une longue tradition sur le plan philosophique et épistémologique (Lenoir, 2017). Cette appréhension du système didactique, qui est un modèle réducteur comme tout modèle, mais qui peut devenir un guide conceptuel éclairant, repose sur une vision dialectique, tripolaire, de l'interaction entre trois éléments constitutifs (empirique, théorique, opératoire) et non pas sur une vision causale traditionnelle d'interrelations entre deux éléments. La limite principale de ce modèle est l'oubli fréquent de son insertion dans le contexte social, ainsi que le relève par exemple Johsua (1999), oubli que nous discutons (Favre et Lenoir, 2015) et que nous le développons longuement (Lenoir, 2017) en nous appuyant sur les travaux de Freitag (1986, 2011). Ce dernier complète les trois fonctions dialectiques traditionnelles (empirique, théorique, opératoire) qui caractérisaient la science moderne par les fonctions de sens (la perspective sociohistorique) et la fonction de valeur (l'ancrage dans le contexte social), fonctions indispensables et incontournables dans le champ éducatif. Autre exemple, le concept de transposition concerne l'aménagement du savoir savant en savoir scolaire de manière à le rendre compréhensible par les élèves. Chevallard (1991) avait proposé le concept de transposition didactique pour désigner le " passage du savoir savant au savoir enseigné, donc à l'éventuelle, à l'obligatoire distance qui les sépare » (p. 15). À ses yeux, ce concept

\footnotetext{
${ }^{26}$ Nous ne pouvons ici que renvoyer aux débats entre les pédagogues, les didacticiens et les disciplinaires. Voir par exemple le n ${ }^{\circ} 137$ de la Revue des sciences de l'éducation (Institut national de recherche pédagogique, 2001), dont le texte présentant les échanges entre Philippe Meirieu et Denis Kamboucher, ou encore Meirieu (1992) sur les attaques de la pédagogie ou Marchive (2008) qui traite des rapports entre ces deux concepts dans l'enseignement, soulignant dans les didactiques une appréhension scientifique des conditions de transmission des connaissances, mais relevant que cette scientificité ne considère que l'aspect cognitif de ce qui se passe dans une classe. Nous avons proposé (Lenoir, 2017) une interaction forte entre processus cognitifs et processus pédagogiques en faisant appel au concept de médiation.
} 
est un outil de vigilance épistémologique et il renvoie pour l'enseignant à «certaines contraintes didactiques spécifiques » (Chevallard, 1991, p. 15) qui obligent à une déformation du savoir tout en assurant une « fiction d'identité ou de conformité acceptable [...]. Le savoir enseigné doit apparaître conforme au savoir à enseigner » (Ibid., p. 16).

Il importe ici de faire remarquer que les curricula de formation au primaire et au secondaire ne sont pas en eux-mêmes le produit directement extrait de disciplines scientifiques; les contenus en sont parfois même totalement étrangers, d'autant plus que leur origine constitutive est diverse (Chervel, 1981, 1988, 1992; Develay, 1992; Goodson, 1981; MesserDavidow, Shumway et Sylvan, 1993; Popkewitz, 1987; Sachot, 1993, 1994) ${ }^{27}$. Les Étatsuniens évitent d'ailleurs cette confusion latente en ayant généralement recours aux termes subject-matter et school subject pour désigner une discipline enseignée : il s'agit d'une matière scolaire. Mais l'affirmation des singularités de chaque matière requiert que la structure conceptuelle commune soit interprétée et réaménagée par chaque didactique en fonction de la spécificité de la discipline à enseigner.

\section{Un coup d'œil ailleurs dans le monde}

La conception francophone de la didactique est-elle le propre de, sinon exclusive à la francophonie? Nous ne le pensons pas, mais nous ne pouvons nous permettre ici que de relever quelques distinctions que nous avons pu retrouver ici et là dans le monde occidental. Notons que la vision présentée est assurément réductrice; elle demanderait autant de nuances que de distinctions plus profondes, tant il existe d'innombrables interprétations.

Dans la perspective étatsunienne d'abord, la recherche anglophone en éducation utilise fort peu le terme didactique. Le plus souvent, il est employé dans un sens péjoratif, celui d'une pratique d'enseignement surannée et hautement ennuyeuse de surcroît. De plus, le Merriam-Webster Dictionary assimile l'adjectif didactic à l'activité pédagogique. Il lui accorde également un sens d'enseignement opposé à celui de divertissement. Par contre, le terme curriculum, à distinguer radicalement de cursus, constitue le concept clef, central et incontournable, avec celui de pédagogie (Lenoir, Hasni et Froelich, 2015). Ces différences ne peuvent se comprendre, ainsi que nous l'avons fait pour les systèmes éducatifs francophone et étatsunien (Lenoir, 2002), que par leur réinsertion et analyse sociohistorique dans la complexité des sociétés dans lesquelles ils se sont constitués et ont

${ }^{27}$ Hasni (2001) procédé à l'analyse de nombreuses publications sur la constitution des disciplines scolaires et il en a dégagé quatre processus de structuration : la discipline scolaire en tant que prolongement de la discipline scientifique ou la dimension disciplinaire; la discipline scolaire en tant que produit et enjeu sociaux ou la dimension sociologique; la discipline scolaire en tant que produit historique de l'école ou la dimension institutionnelle; la discipline scolaire en tant que produit d'une interaction entre la société, l'école et les savoirs scientifiques. Voir également Lenoir et Hasni (2006). 
évolué. Si la notion de didactique se retrouve occasionnellement dans la documentation scientifique étatsunienne, c'est la plupart du temps sous la plume d'auteurs européens (exemple : Tochon, 1999). Un ouvrage comparatif, intitulé Didaktik and/or curriculum. An international dialogue (Gundem et Hopmann, 1998) et des articles de Hopmann (1992) et de Hopmann et Riquarts (1995) avancent l'idée d'un dialogue entre ces deux notions. Il s'agit en fait davantage de discours parallèles !

Dans la tradition germanique plus ancienne, mais aussi scandinave, la conception de la didactique diffère profondément de la conception francophone. On ne peut oublier cependant qu'il existe des différences importantes entre les approches allemande, suédoise, norvégienne et finlandaise (Engelsen, 1990; Englund, 2006; Gundem, 1992, 1995; Gundem et Hopmann, 1998; Hellgren, 1993a, 1993b, 1993c; Hopmann, 1992; Hopmann et Riquarts, 1995; Klafki, 1995; Wallin, 1988; Westbury, Doyle et Künzli, 1993). Elle est conçue soit comme «la mise en place des conditions d'apprentissage en termes de développement psychologique » (Hellgren, 1993b, p. 10), soit comme une méthodologie de l'enseignement (par exemple, Engelsen, 1990; Englund, 2006; Gundem, 1992; Klafki, 1998; Wallin, 1988). Il s'agit, particulièrement en Allemagne, d'une «didactique générale » (allgemeine didaktik) « qui fait clairement partie des sciences de l'éducation et concerne tous les problèmes de l'enseignement et apprentissage d'un point de vue général, indépendamment des disciplines et des contenus » (Dorier, Leutenegger et Schneuwly, 2013, p. 12). Cette conception allemande de la didaktik est évidemment rejetée par les didacticiens français qui ne pensent avant tout qu'en termes de didactique spécifique à chaque discipline d'enseignement.

Dans d'autres pays, par exemple au Brésil et ailleurs en Amérique latine, la didactique est souvent définie, ainsi que le signale Alencastro Veiga $(1988,2002)$ soit comme une simple transmission du savoir, soit comme le travail enseignant, soit comme une méthodologie de l'enseignement, soit souvent comme des processus pédagogiques. Ainsi, Amaya Guerra (2005) considère que « la meilleure technique didactique pour assurer les apprentissages est la manipulation des objets qui favorise la compréhension et la création de la connaissance» (p. 113). Mais elle est aussi porteuse d'une posture critique. Cette perspective, de type politique, de la didactique se retrouve par exemple chez Alencastro Veiga (2002) ou chez Teixeira Damis (1996) qui s'appuie sur Comenius et sur le matérialisme historique pour y voir un processus critique de mise en cause sociale des rapports aux savoirs. Mais elle est également comprise de nos jours comme une théorie de l'enseignement qui implique, en plus d'une dimension politique, une dimension épistémologique (Libâneo, 1990, 2013). 


\section{Conclusion : aux fondements sociohistoriques des conceptions}

Si toutes les différentes interprétations du concept de didactique font appel à des finalités pour en justifier la visée et l'usage, il ressort nettement que la conception francophone, à l'encontre d'autres conceptions, est étroitement associée à la priorité accordée aux savoirs disciplinaires, par là à la problématique du rapport aux savoirs et aux enjeux épistémologiques. Pourquoi en est-il ainsi? De manière très schématique, car il faudrait mener à bien d'amples développements conceptuels ${ }^{28}$ et sociohistoriques - et introduire de nombreuses nuances - pour cerner les raisons qui ont conduit à ces différences de logiques sur le plan éducatif. Nous pouvons toutefois rappeler au moins ce qui suit en ce qui regarde historiquement l'Europe francophone et l'Amérique du Nord anglophone (Lenoir, 2002; Lenoir, Hasni et Froelich, 2015).

Aux États-Unis, en fonction d'une tradition qui s'épanouit dans la deuxième moitié du $19^{\mathrm{e}}$ siècle, la conception du curriculum est centrée sur la mise en œuvre des pratiques enseignantes, sur les questions pédagogiques (le comment faire). C'est ce dont témoigne explicitement Kliebard (1992a) pour qui les modalités opératoires sont prioritaires. Dans la logique américaine, comme le montrent les travaux de différents auteurs étatsuniens (Cremin, 1955, 1964; Franklin, 1986; Kliebard, 1986, 1992a, 1992b; Pinar, Reynolds, Slattery et Taubman, 1995; Rudolph, 1977; Tanner et Tanner, 1990; Willis, Schubert, Bullough, Kridel et Holton, 1993), s'impose à la fin du $19^{\mathrm{e}}$ siècle, à la suite de la guerre de Sécession et avec la vague de l'industrialisation galopante, un nouveau modèle, dont Whitehead (1929) s'est fait entre autres le chantre (Boix-Mansilla et Lenoir, 2010; Lenoir, 2002). Ce nouveau modèle, qui peut être qualifié de pragmatique, est centré sur le savoirfaire. C'est en faisant que le citoyen américain se réalise, s'émancipe. Cette conception, dite vocationaliste, c'est-à-dire professionnalisante, se caractérise par une formation fondée sur le développement des compétences jugées utiles pour répondre aux besoins et aux attentes de la société. Pinar (1998) l'énonce explicitement: « [t]he American public schools were created over 100 years ago to prepare citizens for jobs in an industrial economy » (p. 205). Il s'agit d'un renversement de conception de l'éducation qui constitue, de la part de mouvements progressistes (Parker, Quincy, Ward, Herbartians, Dewey, etc.), une opposition aux conceptions humanistes traditionnelles mettant en avant une éducation non utilitariste, culturelle et académique (Rudolph, 1977) qui découlait de la conception traditionnelle britannique, culturelle et humaniste, de l'éducation dont la figure emblématique était le cardinal Newman (1852/1907; 1872/1909) (Lenoir, 2016), mais

\footnotetext{
${ }^{28}$ Par exemple, les notions de liberté, de république, de citoyenneté, de communauté, etc., se déclinent différemment aux États-Unis et en France (Lenoir, 2002; Lenoir, Adigüzel, Lenoir, Libâneo et Tupin, 2016; Lenoir, Hasni et Froelich, 2015).
} 
aussi, plus profondément de la conception de l'éducation des êtres humains au cours de la Renaissance (d'Irsay, 1933; Freitag, 2011; Garin, 1968).

Aux États-Unis, le souci à l'égard de la fonctionnalité des apprentissages trouve donc ses fondements dans le pragmatisme qui s'instaure aux États-Unis à la fin du $19^{\mathrm{e}}$ siècle. Il importe d'y associer cette préoccupation datant de la même époque qui entend mettre l'élève en tant qu'individu au centre des apprentissages (le pôle sujet). Le besoin d'intégration sociale des nouveaux émigrants arrivant en grand nombre imposait une centration sur les dimensions pédagogiques (pour faciliter les processus d'apprentissage) et les processus de socialisation (le savoir-être citoyen). Ces deux traits fondamentaux de la conception curriculaire nord-américaine soutiennent une orientation professionnalisante de son système éducatif scolaire. Aux États-Unis, la priorité serait accordée à l'adhésion aux vertus et aux valeurs de la société américaine (au savoir-être), ainsi qu'au savoir-faire axé sur la résolution de problèmes. De plus, le débat épistémologique qui sous-tend la sélection des savoirs et la constitution des curricula est postulée avoir été réglé en amont.

À l'opposé, en Europe francophone, la Révolution française de 1789 est marquée par un rationalisme issu des Lumières, par le rejet de toute forme de contrôle extérieur que l'Église a assurée jusqu'au $18^{\mathrm{e}}$ siècle, par la substitution de l'État laïc à la puissance divine. Dans l'esprit des révolutionnaires français, instruire est la responsabilité première de l'Étatnation, car elle est à la source de la liberté humaine et la meilleure assurance pour former un citoyen libre et émancipé, et garantir une société démocratique. L'atteinte de la liberté humaine, par là de l'émancipation, passe dès lors prioritairement par l'instruction, l'acquisition des connaissances que les disciplines scientifiques élaborent et qui sont soutenues par la raison. Dès lors, la formation scolaire a eu pour unique fonction de transmettre de savoirs disciplinaires parce que ce sont, dans la logique francophone, les savoirs qui ouvrent la porte à la liberté humaine. Mais l'instruction ne peut toutefois se réduire, dans la pensée de Condorcet (1971-1972/1989a, 1989b), à la simple transmission de savoirs. L'instruction est libératrice dans la mesure où elle s'appuie sur la raison, sur une démarche rationnelle.

Ainsi que le remarque Sachot (2000), la Révolution française introduit une rupture majeure avec le système éducatif en vigueur jusqu'alors. Ainsi, le rapport au savoir, à la discipline scientifique, s'avère fondamental, car il devient le garant de la tradition culturelle. Il importe donc de problématiser le savoir, d'en interroger le sens avant d'agir, de débattre des contenus cognitifs à soumettre aux apprentissages des futurs citoyens : en conséquence, souligne Sachot, le rapport au savoir est premier. Il importe cependant de se questionner sur ses attributs, sur son caractère scientifique, sur ses apports et de s'assurer que les savoirs sélectionnés sont à la fois exempts des biais idéologiques et qu'ils sont accessibles aux 
élèves. Les perspectives épistémologiques et didactiques viennent assurer ces fonctions. Bref, la recherche du sens et la définition des objets d'enseignement s'avèrent primordiales.

Ainsi, si des deux côtés de l'Atlantique les deux logiques éducatives reposent dès la refondation des systèmes éducatifs scolaires sur la même visée fondamentale, émancipatrice, de l'être humain, elles se sont opposées sur les modalités à mettre en œuvre pour y parvenir. La centration en Europe francophone sur la fonction d'instruction en tant que transmission des savoirs portés par les disciplines et par le patrimoine culturel, a conduit à privilégier la question épistémologique du sens, par là le savoir (le pôle objet) et l'introduction des didactiques des disciplines.

\section{Références}

Abélard (Abaelardi), P. (1851). Sic et non (première édition intégrale sous la direction de E. L. T. Henke et G. S. Lindenkohl). Sumtibus et typis librariae academ. Elwertianae. Aebli, H. (1966). Didactique psychologique. Application à la didactique de la psychologie de Jean Piaget. ( $3^{\mathrm{e}}$ éd.). Delachaux et Niestlé ( $1^{\text {re }}$ éd. 1951).

Alencastro Veiga, I. (dir.). (1988). Repensando a didática. Papirus Editora.

Alencastro Veiga, I. (2002). A prática pedagógica do professor de didática. Papirus Editora.

Amaya Guerra, J. (2005). Fracasos y falacias de la educación actual. Guía para padres y maestros orientando a revalorar lo importante de la educación. Editorial Trillas.

Artigue, M. et Douady, R. (1986). La didactique des mathématiques en France. Émergence d'un champ scientifique. Revue française de pédagogie, 76, 69-88.

Artigue, M., Gras, R., Laborde, C. et Tavignot, P. (1994). Vingt ans de didactique des mathématiques en France. Hommage à Guy Brousseau et Gérard Vergnaud. La Pensée sauvage.

Astolfi, J.-P. (1990). L'émergence de la didactique de la biologie, un itinéraire. Aster, 11, $195-224$.

Astolfi, J.-P. (1997). Du "tout" didactique au "plus" didactique. Revue française de pédagogie, 120, 67-73.

Astolfi, J.-P. et Develay, M. (1989). La didactique des sciences. Presses universitaires de France.

Audigier, F. (1988). Didactique de l'histoire, de la géographie et des sciences sociales: propos introductifs. Revue française de pédagogie, 85, 5-9.

Balacheff, N. et Laborde, J.-M. (1992). Modélisation informatique des processus didactiques. Courrier du CNRS, 79, 69.

Benvéniste, É. (1969). Le vocabulaire des institutions indo-européennes (2 t.). Éditions de Minuit. 
Best, F. (1973). Pour une pédagogie de l'éveil. Bourrelier Éducation/A. Colin.

Best, F. (2006). Croisement(s) du pédagogique et du politique : le cas des activités d'éveil. Les sciences de l'éducation - Pour l'ère nouvelle, 4(39), 47-61.

Bloch, M. (1977). Apologie pour l'histoire ou métier d'historien ( $7^{\mathrm{e}}$ éd.). Armand Colin

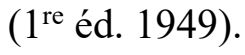

Boix-Mansilla, V. et Lenoir, Y. (2010). Interdisciplinarity in United States schools : Past, present, and future. Issues in Integrative Studies, 28, 1-28.

Bronckart, J.-P. (1989). Du statut des didactiques des matières scolaires. Langue française, $82,53-66$.

Bronckart, J.-P. et Schneuwly, B. (1991). La didactique du français langue maternelle : l'émergence d'une utopie indispensable. Bildungsforschung und Bildungspraxis/Éducation et recherche, 13(1), 8-25.

Brousseau, G. (1986). Fondements et méthodes de la didactique des mathématiques. Recherches en didactique des mathématiques, 7(2), 33-115.

Brousseau, G. (2004). L'émergence d'une science de la didactique des mathématiques. Repères IREM, 55, 19-34.

Bru, M. (2006). Les méthodes en pédagogie. Presses universitaires de France.

Canavolas, J. A. et Bilbeau, G. (1996). Jan Amos Coménius (1592-1670). Aspects culturels, philosophiques, pédagogiques et didactiques de son ouvre (Actes du colloque international Coménius). Université de Montréal, Faculté des sciences de l'éducation.

Capková, D. (1992). La conception de la "Cultura universalis" chez Coménius. Dans J. A. Caravolas et G. Bibeau (dir.), Jean Amos Coménius (1592-1670). Aspects culturels, philosophiques, pédagogiques et didactiques de son œuvre (Actes du colloque international Coménius (p. 19-39). Université de Montréal, Faculté des sciences de l'éducation.

Cauly, O. (1995). Comenius. Éditions du Félin.

Chené, A., Gauthier, C., Martineau, S., Dolbec, A., Lenoir, Y., Gaudet, J. et Charland, J.P. (1999). Les objets actuels de la recherche en éducation. Revue des sciences de l'éducation, XXV(2), 401-437.

Chervel, A. (1981) ... et il fallut apprendre à écrire à tous les petits Français. Histoire de la grammaire scolaire. Payot.

Chervel, A. (1988). L'histoire des disciplines scolaires. Réflexions sur un domaine de recherche. Histoire de l'éducation, 38, 59-119.

Chervel, A. (1992). L'école, lieu de production d'une culture. Dans F. Audigier et G. Baillat (dir.), Didactiques de l'histoire, de la géographie, des sciences sociales. Analyser et gérer les situations d'enseignement-apprentissage (Actes du sixième colloque, mars 1991, p. 195-198). Institut national de recherche pédagogique.

Chevallard, Y. (1985). La transposition didactique. La pensée sauvage. 
Chiss, J.-L. (1985). Quel statut pour la linguistique dans la didactique du français? Études de linguistique appliquée, 59, 7-16.

Chiss, J.-L. (1989). Revendication d'autonomie et horizon de scientificité en didactique du français. Langue française, 82, 44-52.

Comenius, J. A. (1952). La grande didactique. Traité de l'art universel d'enseigner tout à tous (trad. par J. B. Piobetta). Presses universitaires de France ( $1^{\text {re }}$ éd. 1657).

Condorcet (M. J. A. Caritat, marquis de) (1989a). Écrits sur l'instruction publique. Vol. I : Cinq mémoires sur l'instruction publique (Texte présenté, annoté et commenté par

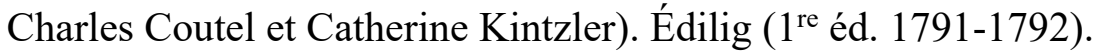

Condorcet (M. J. A. Caritat, marquis de) (1989b). Écrits sur l'instruction publique. Vol. II : Rapport sur l'instruction (Texte présenté, annoté et commenté par Charles Coutel

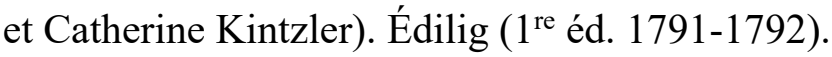

Cornu, L. et Vergnioux, A. (1992). La didactique en questions. Hachette Éducation.

Cremin, L. (1955). The revolution in american secondary education, 1893-1918. Teachers College Record, 56, 295-308.

Cremin, L. (1964). The transformation of the school: Progressivism in american education, 1876-1957. Vintage.

de Caussade, F. (1719). Rhétorique et genres littéraires ( $2^{\mathrm{e}}$ éd. revue). G. Masson, éditeur.

De Corte, E., Geerligs, C. T., Lagerweij, N. A. J., Peters, J. J. et Vandenberghe, R. (1979). Les fondements de l'action didactique. De la didactique à la didaxologie (trad. par

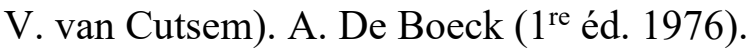

Delumeau, J. (1965). Naissance et affirmation de la Réforme. Presses universitaires de France.

d'Irsay, S. (1933). Histoire des universités françaises et étrangères des origines à nos jours. Tome 1 : Moyen Age et Renaissance. Éditions Auguste Picard.

de Maumont, J. (1554). Les æeuvres de S. Justin philosophe et martyr, mises de grec en françois par Jean de Maumont. Les traictez dudict autheur qui se trounent aujourdhuy, lesquelz ont esté prins de la librairie du feu roy François, sont escripts \& nommez cy apres en la page qui suit le Prologue. Paris : Imprimerie de Michel de Vascosan. Document disponible à l'adresse http://www.sudoc.abes.fr/xslt//DB=2.1/ $\underline{\mathrm{SET}}=3 / \mathrm{TTL}=1 / \mathrm{SHW}$ ?FRST $=1$

Denis, M. (1994). Comenius. Une pédagogie à l'échelle de l'Europe. Presses universitaires de France.

de Saint-Victor, H. (1961). Didascalicon (trad. du latin par J. Taylor). Columbia University Press (1 $1^{\text {re }}$ éd. av. 1137).

Desvé, C. (dir.). (1993). Guide bibliographique des didactiques. Des ressources pour les enseignants et les formateurs. Institut national de recherche pédagogique.

Develay, M. (1992). De l'apprentissage à l'enseignement. Pour une épistémologie scolaire. ESF. 
Develay, M. (1997). Origines, malentendus et spécificités de la didactique. Revue française de pédagogie, 120, 59-66.

Dienes, Z. P. (1964). La mathématique moderne dans l'enseignement primaire. OCDL.

Dorier, J.-C., Leutenegger, F. et Schneuwly, B. (2013). Introduction. Le didactique, les didactiques, la didactique. Dans J.-C. Dorier, F. Leutenegger et B. Schneuwly (dir.), Didactique en construction, construction des didactiques (p. 7-35). De Boeck.

Engelsen, B. U. (1990). Didactics : Swedish and norwegian understandings compared by a Norwegian. Scandinavian Journal of Educational Research, 34(4), 285-299.

Englund, T. (2006). New trends in swedish educational research. Scandinavian Journal of Educational Research, 50, 383-396.

Favre, D. et Lenoir, Y. (2015). La didactique entre simplification et prise en compte de la complexité (étude préliminaire). Éditions universitaires européennes.

Franklin, B. M. (1986). Building the american community: The school curriculum and the search for control. Falmer.

Freitag, M. (1986). Dialectique et société. T. 1 : Introduction à une théorie générale du symbolique. L'âge d'homme/Éditions coopératives Albert Saint-Martin.

Freitag, M. (2011). L'abîme de la liberté. Critique du libéralisme. Liber.

Galisson, R. (1985). Didactologies et idéologies. Études de linguistique appliquée, 60, 516.

Galisson, R. (1986). Éloge de la didactologie/didactique des langues et des cultures (maternelles et étrangères). Études de linguistique appliquée, 64, 39-54.

Galisson, R. (dir.). (1990). De la linguistique appliquée à la didactologie des languescultures. Vingt ans de réflexions disciplinaires. Études de linguistique appliquée, 79 (Numéro thématique).

Garin, É. (1968). L'éducation de l’homme moderne. La pédagogie de la Renaissance (1400-1600). Fayard.

Goodson, I. F. (1981). Becoming an academic subject: Patterns of explanation and evolution. British Journal of Sociology of Education, 2(2), 163-180.

Gundem, B. B. (1992). Notes on the development of nordic didactics. Journal of Curriculum Studies, 24(1), 61-70.

Gundem, B. B. (1995). The role of didactics in curriculum in Scandinavia. Journal of Curriculum and Supervision, 10(4), 302-316.

Gundem, B. B. et Hopmann, S. (dir.). (1998). Didaktik and/or curriculum. An international dialogue. Peter Lang.

Habermas, J. (1973). La technique et la science comme “idéologie” (trad. par J.-R. Ladmiral). Gallimard ( $1^{\text {re }}$ éd. 1968).

Habermas, J. (1976). Connaissance et intérêt (trad. par G. Clémençon). Gallimard (1 $1^{\text {re éd. }}$ 1968). 
Halbwachs, F. (1971). Réflexions sur la causalité physique. Dans M. Bunge, F. Halbwachs, T. S. Kuhn, J. Piaget et L. Rosenfeld, Les théories de la causalité (p. 19-38). Presses universitaires de France

Halté, J.-F. (1992). La didactique du français. Presses universitaires de France.

Hasni, A. (2001). Les représentations sociales d'une discipline scolaire - l'activité scientifique - et sa place au sein des autres disciplines formant le curriculum chez des instituteurs marocains. Thèse de doctorat en éducation, Université de Sherbrooke.

Hellgren, P. (1993a). What is the epistemological identity of subject-specific didactics in Finland? Dans L. Montero Mesa et J. M. Vez Jeremias (dir.), Las didácticas especificas en la formación del profesorado (p. 571-577). Tórculo Ediciónes.

Hellgren, P. (1993b). The dialectic of school curriculum and teacher education. Texte de la communication lors du $13^{\mathrm{e}}$ Annual International Seminar for Teacher Education (ISTE), Steinbach-Comburg, Germany, 21-23 avril.

Hellgren, P. (1993c). Developing academic professionals through the finnish teacher education curriculum. Paper presented at the Annual Meeting of the American Educational Research Association (AERA), San Francisco, CA, 20-24 avril.

Hopmann, S. (1992). Starting a dialogue. Roots and issues of the beginning conversation between european didaktik and the american curriculum tradition. Communication présentée au congrès annuel de 1'American Educational Research Association, San Francisco.

Hopmann, S. et Riquarts, K. (1995). Starting a dialogue : Roots and issues in the beginning conversation between the didaktik and curriculum traditions. Journal of Curriculum Studies, 27, 3-12.

Houssaye, J. (1988). Théorie et pratique de l'éducation scolaire. T. 1 : Le triangle pédagogique. Peter Lang.

Houssaye, J. (1994). Le triangle pédagogique, ou comment comprendre la situation pédagogique. Dans J. Houssaye (dir.), La pédagogie: une encyclopédie pour aujourd'hui (p. 13-24). ESF (1 $1^{\text {re }}$ éd. 1993).

Houssaye, J. (1997). Spécificité et dénégation de la pédagogie. Revue française de pédagogie, 120, 83-97.

Institut national de recherche et de documentation pédagogiques (1971). Les activités d'éveil à dominante intellectuelle au cours préparatoire. Recherches pédagogiques, 51, 1-154.

Institut national de recherche et de documentation pédagogiques (1973). Activités d'éveil à l'école élémentaire. I : Objectifs - Méthodes - Moyens. Recherches pédagogiques, 62, 1-249. 
Institut national de recherche et de documentation pédagogiques (1974). Activités d'éveil scientifiques à l'école élémentaire. Première approche de problèmes écologiques. Recherches pédagogiques, 70, 1-307.

Institut national de recherche et de documentation pédagogiques (1978). Activités d'éveil. Sciences sociales à l'élémentaire. Recherches pédagogiques, 93, 1-321.

Institut national de recherche pédagogique (2001). La pédagogie et les savoirs : éléments de débat. Revue des sciences de l'éducation, 137, 5-116.

Johsua (1999). Pour une didactique sociologique (Entretien avec Samuel Johsua, professeur à l'Université d'Aix-Marseille II, didacticien des sciences et des mathématiques). Éducation et sociétés, 4, 29-56.

Jonnaert, P. (1991a). Didactique : évolution d'un concept, naissance d'une discipline. Pédagogies, 1, 97-111.

Jonnaert, P. (1991b). Didactique et solidarité fonctionnelle de trois familles de variables. Dans P. Jonnaert (dir.), Les didactiques. Similitudes et spécificités (p. 11-25). Plantyn.

Klafki, W. (1998). Characteristics of critical-constructive didaktik. Dans B. B. Gundem et S. Hopmann (dir.), Didaktik and/or curriculum (p. 307-330). Peter Lang.

Kliebard, H. M. (1986). The struggle for the american curriculum : 1893-1958. Routledge \& Kegan Paul.

Kliebard, H. M. (1992a). Forging the american curriculum. Essays in curriculum history and theory. Routledge.

Kliebard, H. M. (1992b). Constructing a history of the american Curriculum. Dans P. H. Jackson (dir.), Handbook of research on curriculum. A project of the American Educational Research Association (p. 157-184). Macmillan.

Lebrun, J. et Lenoir, Y. (2004). La conception de la didactique des sciences humaines dans la documentation scientifique française. Dans M. Sachot et Y. Lenoir (dir.), Les enseignants du primaire entre disciplinarité et interdisciplinarité : quelle formation didactique? (p. 133-165). Presses de l'Université Laval.

Legendre, R. (1983). L'éducation totale. F. Nathan/Ville-Marie.

Lemerle, P. (1971). Le premier humanisme byzantin. Notes et remarques sur enseignement et culture à Byzance des origines au $X^{e}$ siècle. Presses universitaires de France.

Le Moigne, J.-L. (1984). La théorie du système général. Théorie de la modélisation. Presses universitaires de France.

Lemoyne, G. (1996). La recherche en didactique des mathématiques au Québec: rétrospectives et perspectives. Bulletin $A M Q, X X X V I(3), 31-40$.

Lenoir, Y. (1993). Entre Hegel et Descartes : de quels sens peut-il être question en didactique? Dans P. Jonnaert et Y. Lenoir (dir.), Sens des didactiques et didactique du sens (p. 29-99). Éditions du CRP. 
Lenoir, Y. (2000). La recherche dans le champ des didactiques : quelques remarques sur les types de recherches, leur pertinence et leurs limites pour la formation à l'enseignement. Revue suisse des sciences de l'éducation, 22(1), 177-222.

Lenoir, Y. (2002). Les réformes actuelles de la formation à l'enseignement en France et aux États-Unis : éléments de mise en perspective socio-historique à partir du concept d'éducation. Revue suisse des sciences de l'éducation, 24(1), 91-128.

Lenoir, Y. (2011). Fondements épistémologiques et cadre de référence des travaux de recherche de la Chaire de recherche du Canada sur l'intervention éducative. Dans $\mathrm{C}$. Day et C. Laneve (dir.), Analysis of educational practices. A comparison of research models (p. 59-82). Editrice La Scuola

Lenoir, Y. (2016). Les finalités éducatives : une illustration d'une évolution de plusieurs conceptions directement influencées par les fondements socio-philosophiques. Dans Y. Lenoir, O. Adiguzel, A. Lenoir, J. C. Libâneo et F. Tupin (dir.), Les finalités éducatives scolaires. Pour une étude critique des approches théoriques, philosophiques et idéologiques. T. 1 : Fondements, notions et enjeux socioéducatifs (p. 255-282). Éditions Cursus universitaire.

Lenoir, Y. (2017). Les médiations au cour des pratiques d'enseignement-apprentissage : une approche dialectique. Des fondements à leur actualisation en classe. Éléments pour une théorie de l'intervention éducative ( $2^{\mathrm{e}} \mathrm{e} d$. rev. et augm.). Éditions Cursus universitaire ( $1^{\text {re }}$ éd. 2014).

Lenoir, Y. (2019a). Quelques fondements pour caractériser l'intervention éducative dans le cadre des processus d'enseignement-apprentissage. Revue des sciences de l'éducation, 44(3), 159-189.

Lenoir, Y (2019b). Praxis et pragmatisme utilitariste dans les rapports entre action et connaissance en éducation : dualisme ou tension féconde? Dans V. Bedin, S. Franc et D. Guy (dir.), Les sciences de l'éducation pour quoi faire? Entre action et connaissance (p. 57-75). L'Harmattan.

Lenoir, Y., Adigüzel, O., Lenoir, A., Libâneo, J. C. et Tupin, F. (dir.). (2016). Les finalités éducatives scolaires. Une étude critique des approches théoriques, philosophiques et idéologiques. T. 1 : Fondements, notions et enjeux socioéducatifs. Éditions Cursus universitaire.

Lenoir, Y., Esquivel, R. et Jean, V. (2020). Une recherche comparative internationale sur les finalités éducatives scolaires: clarifications conceptuelles et caractéristiques. Dans Y. Lenoir, J. Bourque, A. Hasni, R. Nagy et M. Priolet, M. (dir.), Les finalités éducatives scolaires. Pour une étude critique des approches théoriques, philosophiques et idéologiques. T. 2 : Conceptions des finalités et des disciplines scolaires chez des enseignants du primaire. Une étude comparative internationale (p. 17-35). Éditions Cursus universitaire. 
Lenoir, Y. et Hasni, A. (2006). Les disciplines, la didactique des disciplines et le curriculum de formation à l'enseignement primaire : de la maîtrise à l'adéquation. Dans Y. Lenoir et M.-H. Bouillier-Oudot (dir.), Savoirs professionnels et curriculum de formation (p. 125-166). Presses de l'Université Laval.

Lenoir, Y., Hasni, A. et Froelich, A. (2015). Curricular and didactic conceptions of interdisciplinarity in the field of education : A socio-historical perspective. Issues in Interdisciplinary Studies, 33, 39-93.

Lenoir, Y., Rey, B. et Fazenda, I. (dir.). (2001). Les fondements de l'interdisciplinarité dans la formation à l'enseignement. Éditions du CRP.

Lenoir, Y. et Sauvé, L. (1998a). De l'interdisciplinarité scolaire à l'interdisciplinarité dans la formation à l'enseignement: un état de la question. 1 - Nécessité de l'interdisciplinarité et rappel historique. Revue française de pédagogie, 124, 121153.

Lenoir, Y. et Sauvé, L. (1998b). De l'interdisciplinarité scolaire à l'interdisciplinarité dans la formation à l'enseignement : un état de la question. 2 - Interdisciplinarité scolaire et formation interdisciplinaire à l'enseignement. Revue française de pédagogie, 125, $109-146$.

Libâneo, J. C. (2000). Didática. Cortez.

Libâneo, J. C. (2013). Didática como campo investigativo e disciplinar e seu lugar na formação de professores no Brasil. Dans M. R. S. Oliveira et J. A. Pacheco (dir.), Currículo, didática e formação de professores (p. 131-166). Papirus Editora.

Marchive, A. (2008). La pédagogie à l'épreuve de la didactique: approche historique, perspectives théoriques et recherches empiriques. Presses universitaires de Rennes.

Margolinas, C. (1993). De l'importance du vrai et du faux dans la classe de mathématiques. La Pensée sauvage.

Marquez, A. D. (1960). Idées pour une didactique opératoire. Revue belge de psychologie et de pédagogie, XXII(89), 1-10.

Marrou, H.-I. (1965). Histoire de l'éducation dans l'Antiquité (6éd. revue et augmentée).

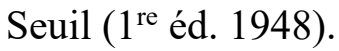

Meirieu, P. (1985). L'école mode d'emploi. Des "méthodes actives" à la pédagogie différenciée. ESF.

Meirieu, P. (1987). Apprendre... oui, mais comment? ESF.

Meirieu, P. et Develay, M. (1992). Émile, reviens vite... ils sont devenus fous. ESF.

Messer-Davidow, E., Shumway, D. R. et Sylvan, D. J. (1993). Knowledges: Historical and critical Studies in disciplinarity. University Press of Virginia.

Mialaret, G. (dir.). (1979). Vocabulaire de l'éducation. Presses universitaires de France.

Morf, A. (1972). La formation des connaissances et la théorie didactique. Dialectica, 26(2), $103-114$. 
Morf, A., Grize, J.-B. et Pauli, L. (1969). Vers une pédagogie scientifique. Dialectica, 23(1), 24-31.

Morin, E. (1977). La méthode. T. 1 : La nature de la nature. Seuil.

Morin, E. (1980). La méthode. T. 2 : La vie de la vie. Seuil.

Moscovici, S. (1970). Préface. Dans D. Jodelet, J. Viet et P. Besnard (dir.), La psychologie sociale, une discipline en mouvement (p. 9-64). Maloine/Mouton.

Nau, F. (1902). La didascalie c'est-à-dire L'enseignement catholique des douze apôtres et des saints disciples de notre Sauveur traduite du syriaque par F. Nau. Lethielleux, Libraire-éditeur.

Newman, J. H. (1907). The idea of University: Defined and illustrated. Longmans, Green and Co (1 ${ }^{\text {re }}$ éd. 1852). Document accessible à l'adresse http://www.newmanreader.org/works/idea/index.html\#contents

Newman, J. H. (1909). Historical sketches. Vol. 3 : Rise and progress of universities. Longmans, Green and Co ( $1^{\text {re }}$ éd. 1872). Document accessible à l'adresse http://www.newmanreader.org/works/historical/volume3/index.html\#titlepage

Paquette, C. (1976). Vers une pratique de la pédagogie ouverte. NHP.

Paquette, C. (1985). Pédagogie ouverte et autodéveloppement. NHP.

Paré, A. (1977). Créativité et pédagogie ouverte. T. 1 : Pédagogie encyclopédique et pédagogie ouverte. T. 2 : Créativité et apprentissage. T. 3 :Organisation de la classe et intervention pédagogique. NHP.

Paré, G., Brunet, A. et Tremblay, P. (1933). La renaissance du XII siècle. Les écoles et l'enseignement. Librairie philosophique J. Vrin/Institut d'études médiévales.

Piaget, J. (1967). Les courants de l'épistémologie scientifique contemporaine. Dans J. Piaget (dir.), Logique et connaissance scientifique (p. 1225-1271). Gallimard.

Piaget, J. (1971). La causalité selon E. Meyerson. Dans M. Bunge, F. Halbwachs, T. S. Kuhn et L. Rosenfeld, Les théories de la causalité (p. 151-208). Presses universitaires de France.

Pinar, W. F. (1998). Dreamt into existence by others: Notes on school reform in the US. Dans M. Tardif, C. Lessard et C. Gauthier (dir.), Formation des maîtres et contextes sociaux. Perspectives internationales (p. 201-229). Presses universitaires de France.

Pinar, W. F., Reynolds, W. M., Slattery, P. et Taubman, P. M. (1995). Understanding curriculum. An introduction to the study of historical and contemporary curriculum discourses. Peter Lang.

Popkewitz, T. S. (1987). The formation of school subjects. The struggle for creating an american institution. The Falmer Press.

Raisky, C. (1996). Doit-on en finir avec la transposition didactique? Essai de contribution à une théorie didactique. Dans C. Raisky et M. Caillot (dir.), Au-delà des didactiques, le didactique. Débats autour de concepts fédérateurs (p. 37-59). De Boeck Université. 
Reuter, Y. (2010). Dictionnaire des concepts fondamentaux des didactiques. De Boeck.

Rudolph, F. (1977). Curriculum. A history of the american undergraduate course of study since 1636. Jossey-Bass.

Sachot, M. (1993). La notion de "dicipline scolaire" : éléments de constitution. Dans J.-P. Clément, M. Herr et P. Boyer (dir.), L'identité de l'éducation physique scolaire au XXe siècle : entre l'école et le sport (p. 127-147). Éditions AFRAPS.

Sachot, M. (1994). Essai de typologie des disciplines. Communication à la Biennale de l'éducation et de la formation, 9-12 avril, La Sorbonne, Paris.

Sachot, M. (2000). La formation professionnelle à l'enseignement entre deux vecteurs intégrateurs : les disciplines et le curriculum. Éducation et francophonie, XXVIII(2). Document accessible à l'adresse http://www.acelf.ca/revue/

Schneuwly, B. (1990a). Didactique : quelques notes sur son histoire. La lettre de la DFLM, 7, 22-24.

Schneuwly, B. (1990b). Didaktik/Didactiques. Éducation et recherche, 12(3), 213-220.

Segond, L. (1880). La Sainte Bible. Imprimerie de l'Université. Document accessible à l'adresse http://levigilant.com/Bible_Segond_1880_virtuelle/

Serri, J.-P. (1977). Les activités d'éveil. Delagrave.

Servier, J. (1967). Histoire de l'utopie. Gallimard.

Simoneaux, L. et Simoneaux, J. (2014). The emergence of recent science education research and its affiliations in France. Perspectives in Science, 2(1-4), 55-64.

Shorter, E. (1977). Naissance de la famille moderne, XVIII ${ }^{e}$ XXe siècle. Seuil.

Snyders, G. (1965). La pédagogie en France aux XVII et XVIII ${ }^{e}$ siècles. Presses universitaires de France.

Tanner, D. et Tanner, L. (1990). History of the school curriculum. Macmillan/Collier Macmillan.

Tasra, S. (2017). Pédagogie, didactique générale et didactique disciplinaire. Document accessible à l'adresse https://halshs.archives-ouvertes.fr/halshs-01531812/document

Teixeira Damis, O. (1996). Didática e sociedade : o conteúdo implícito do ato de ensinar. Dans I. Alencastro Veiga, I. (dir.), Didática : o ensino e suas relações (p. 9-31). Papirus Editora.

Tochon, F. (1999). Didactics in the francophone world. Instructional Science, 1-4, 1-192.

Vergnaud, G. (dir.). (1994). Apprentissages et didactiques, où en est-on? Hachette Éducation.

Vigour, C. (2005). La comparaison dans les sciences sociales. Pratiques et méthodes. La découverte.

Wallin, E. (1988). Notes on didactics as a field of research. Scandinavian Journal of Educational Research, 32(1), 1-7.

Westbury, I., Doyle, W. et Künzli, R. (1993). The german didactic tradition : Implications for pedagogical research. University of Illinois at Urbana-Champaign. 
Whitehead, A. N. (1929). The aims of education. Williams and Norgate.

Willis, G., Schubert, W., Bullough, R., Kridel, C. et Holton, J. (dir.). (1993). The american curriculum : A documentary history. Greenwood. 